$01 ; 11$

\title{
Взаимодействие химерных состояний в многослойной сети нелокально связанных осцилляторов
}

\author{
(C) М.В. Горемыко ${ }^{1}$, В.А. Максименко ${ }^{1}$, В.В. Макаров ${ }^{1}$, Д. Гош ${ }^{2}$, \\ Б. Бера ${ }^{2}$, C.К. Дана ${ }^{3}$, А.Е. Храмов ${ }^{1, \uparrow}$ \\ ${ }^{1}$ Саратовский государственный технический университет \\ им. Гагарина Ю.А., Саратов, Россия \\ ${ }^{2}$ Индийский статистический институт, Калькутта, Индия \\ ${ }^{3}$ Индийский институт химической биологии, Калькутта, Индия \\ 『E-mail: hramovae@gmail.com
}

Поступило в Редакцию 28 апреля 2017 г.

Изучены процессы возникновения и эволюции химерных состояний в модели многослойной сети нелинейных элементов со сложной топологией связей. В качестве объекта исследования рассмотрена двухслойная сеть нелокально связанных в пределах слоя фазовых осцилляторов Курамото-Сакагучи. Продемонстрированы различные режимы, реализующиеся в данной системе при изменении степени взаимодействия между слоями сети.

DOI: $10.21883 /$ PJTF.2017.15.44871.16270

В настоящее время в радиофизике, биофизике и нелинейной динамике наблюдается значительный интерес к изучению химерных состояний [1], реализующихся в ансамблях нелинейных осцилляторов различной природы. Подобные состояния, характеризующиеся сосуществованием в сетях связанных осцилляторов групп когерентных и некогерентных элементов, были впервые обнаружены в 2002 г. [2] в сети нелокально связанных нелинейных элементов, описываемых уравнением типа Гинзбурга-Ландау.

В последнее десятилетие был опубликован ряд работ, описывающих реализацию химерных состояний в сетях нелинейных элементов, характеризующихся различным типом динамики. В данном контексте было рассмотрено образование химерных состояний как в одномерных системах (цепочках связанных осцилляторов) [3], так и в распределенных системах, состоящих из эквидистантно расположенных осцилляторов, 
описывающихся уравнениями Ресслера [4], Фитцхью-Нагумо [5] и др. Химерные состояния были обнаружены в сетях с различной топологией связей. В частности, в работе [6] на примере сети нейронов ХиндмаршРоуз химерные состояния были получены для случаев глобальной, нелокальной и даже локальной связи. Помимо симметричных связей, в работе [7] также была продемонстрирована возможность реализации химерных состояний в свободно масштабируемой (англ. scale-free) сети. Наряду с анализом модельных систем, интерес к химерным состояниям также обусловлен обнаружением в реальных системах состояний, свойства которых соответствуют химерам. В качестве таких систем, как правило, рассматриваются объекты биологической, химической и электронной природы [8-10].

Среди большого числа эффектов, ассоциирующихся с возникновением химерных состояний, важным и практически неизученным является вопрос устойчивости последних [11], в том числе в случае взаимодействия системы, демонстрирующей химеру, с системой, характеризующейся когерентным поведением всех узлов или полной некогерентностью. Очевидно, что подобная ситуация является распространенной в реальных системах, относящихся к соответствующим областям науки (например, нейронауки [12,13]), и ее рассмотрение, наряду с теоретическим интересом, обусловлено перспективой практического использования для понимания процессов в нейронных сетях [14].

Наиболее подходящей математической и физической моделью для изучения процессов взаимодействия объектов с сетевой структурой является модель многослойной сети (англ. multilayer network). Данная модель характеризуется наличием у каждого элемента двух типов связей. Первый тип характеризует взаимодействие элемента с другими узлами сети, находящимися в пределах одного слоя. Второй тип определяет связь данного элемента с элементами, относящимися к другим слоям сети. В зависимости от специфики задачи конфигурации связей между элементами многослойной сети могут быть различными. В рамках нашей работы будет рассмотрена конфигурация, описанная в нашей недавней работе [15].

Согласно [15], сеть, состоящая из $N^{M}$ элементов, представляется в виде набора из $M$ слоев (по $N$ элементов на каждом слое). Связи между элементами внутри слоя распределены нелокально [6] (каждый элемент связан с $2 R$ соседними элементами), взаимодействие между слоями реализуется посредством локальных связей между двумя смежными 


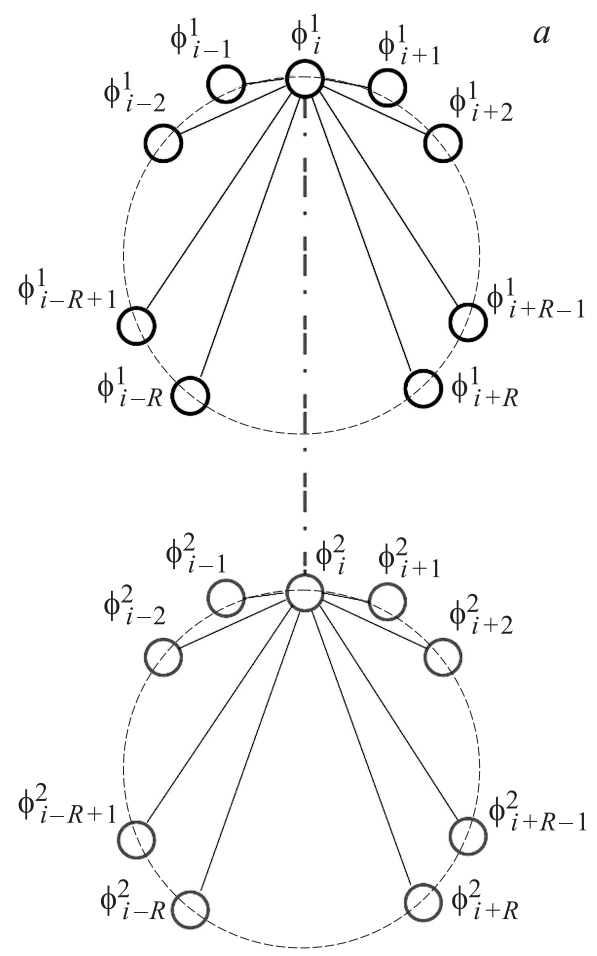

Рис. 1. Схематическое изображение модели многослойной сети с нелокальной связью между элементами одного слоя $(a)$, распределения фаз осцилляторов на слоях в случае отсутствия межслойной связи $(b)$, зависимость степени некогерентности $S_{I}$ от фазового сдвига $\alpha(c)$. Осцилляторы первого слоя обозначены символами + , второго слоя - символами $\times$.

элементами. Схематически подобная модель изображена на рис. $1, a$. Величины $\phi_{i}^{j}$ соответствуют динамическим переменным, характеризующим состояние узла сети, индексы $i$ и $j$ соответствуют порядковому номеру элемента внутри слоя и номеру слоя соответственно. Сплошными линиями обозначены связи элемента $\phi_{i}^{j}$ с соседними элементами внутри слоя, штрихпунктирной линией - межслойная связь, реализованная через взаимодействие элемента первого слоя $\phi_{i}^{1}$ со смежным элементом второго слоя $\phi_{i}^{2}$.

Письма в ЖТФ, 2017, том 43, вып. 15 

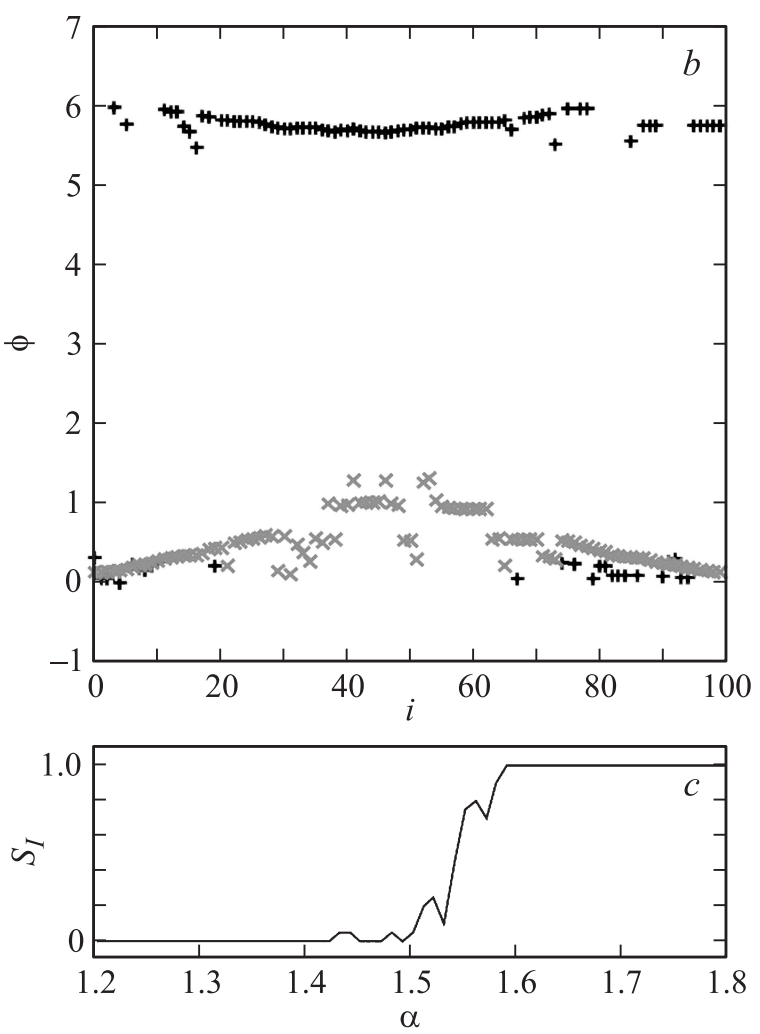

Рис. 1 (продолжение).

Для моделирования динамики узла сети в настоящей работе используется фазовый осциллятор Курамото-Сакагучи (1) [16], который часто применяется как базовая модель для численного и аналитического исследования химерных состояний [17]:

$$
\frac{d \varphi_{i}^{j}}{d t}=\omega_{i}^{j}-\frac{\lambda_{1}}{2 R+1} \sum_{k=i-2 R}^{i+2 R} \sin \left(\varphi_{i}^{j}-\varphi_{k}^{j}+\alpha\right)+\frac{\lambda_{2}}{M} \sum_{l \neq j} \sin \left(\varphi_{i}^{j}-\varphi_{i}^{l}\right) .
$$

Здесь $\omega_{i}^{j}-$ собственная частота осциллятора, $\lambda_{1}-$ коэффициент связи между осцилляторами внутри слоя, $\lambda_{2}-$ коэффициент межслойной 
связи, $R$ - радиус связи, $M-$ число слоев, $\alpha-$ постоянный фазовый сдвиг. В работе рассматривается сеть идентичных осцилляторов $\omega_{i}^{j}=1, \forall i, j$. При этом значения параметров выбраны как $\lambda_{1}=0.085, N=100, M=2, R=35$. Следует отметить, что характеристики осцилляторов и топология связей внутри исследуемых слоев сети являются одинаковыми и удовлетворяют условию возникновения химерного состояния [17].

Для реализации качественно различных состояний на слоях сети в работе используются различные конфигурации начальных распределений фаз $\phi_{i}^{1}(0) \neq \phi_{i}^{2}(0), \forall i \in[1, N]$. Для использованных начальных условий в случае, когда связь между слоями отсутствует, реализующиеся состояния показаны на рис. $1, b$. Видно, что как на первом, так и на втором слое часть осцилляторов пребывает в когерентном состоянии, в то время как остальные - некогерентны. При этом легко видеть, что порядковые номера осцилляторов, задействованных в когерентных и некогерентных кластерах, на различных слоях не коррелируют между собой.

Для изучения процессов взаимодействия между данными химерными состояниями в работе проводится численное моделирование динамики системы при увеличении параметра межслойной связи $\lambda_{2}$. При этом для количественной диагностики химерного состояния рассчитывается величина $S_{I}(2)$, характеризующая степень некогерентности (англ. strength of incoherent). Для расчета данной величины исследуемый ансамбль осцилляторов разбивается на $m$ групп элементов по $n$ элементов в группе. Для данного ансамбля $S_{I}$ определяется как

$$
S_{I}=1-\frac{\sum_{r=1}^{m} \Theta\left(\delta-\sigma_{r}\right)}{m},
$$

где $\Theta(\cdot)$ - функция Хевисайда, $\sigma$ - описывает стандартное отклонение (3), характеризующее осцилляторы в группе с индексом $r$, $\delta=0.035$ - пороговое значение. Величина $\sigma_{r}$ рассчитывается для каждой группы при помощи соотношения

$$
\sigma_{r}=\left\langle\sqrt{\frac{1}{n} \sum_{s=n(r-1)+1}^{m}\left[\varphi_{s}-\Phi\right]^{2}}\right\rangle,
$$

Письма в ЖТФ, 2017, том 43, вып. 15 


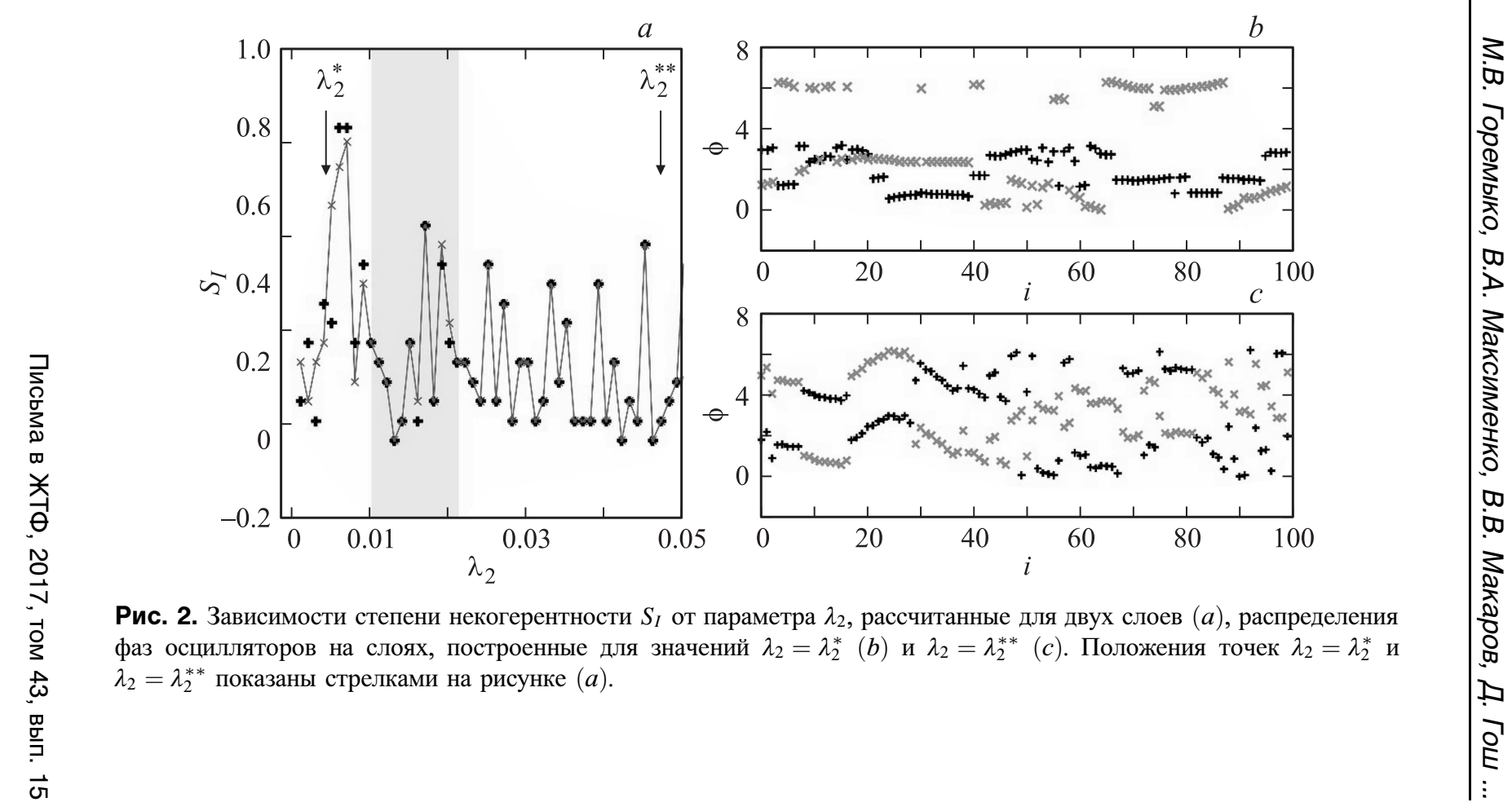


где $\langle\cdot\rangle$ обозначает усреднение по временно́му интервалу, $\Phi-$ средняя фаза по ансамблю.

В зависимости от значения величины $S_{I}$ состояние сети можно трактовать как полностью когерентное $S_{I}=0$, полностью некогерентное $S_{I}=1$ или химерное $0<S_{I}<1$, соответствующее сосуществованию когерентного и некогерентного кластеров.

На рис. 1, $c$ показана зависимость величины $S_{I}$ от величины $\alpha$, характеризующей фазовое соотношение в модели Курамото-Сакагучи. Область параметров, соответствующая химерным состояниям, выделена штриховкой. Исходя из приведенной зависимости, в нашем исследовании значение параметра $\alpha$ для каждого слоя было выбрано как $\alpha=1.45$.

На рис. 2, a приведены зависимости параметра $S_{I}$, рассчитанные для двух слоев рассматриваемой сети при увеличении параметра $\lambda_{2}$. Видно, что при $\lambda_{2}<0.01$ на слоях реализуются химерные состояния с различными свойствами. Типичные распределения фаз осцилляторов для этого случая показаны для двух слоев на рис. 2, $b$. Можно видеть, что как на первом, так и на втором слое существуют когерентные и некогерентные кластеры. Однако фазовые соотношения внутри этих кластеров и, более того, количество элементов, вовлеченных в них, различаются на разных слоях сети. В случае, когда значение $\lambda_{2}$ находится в интервале $[0.01,0.02]$, в сети возможна реализация как состояний, характеризующихся одинаковыми распределениями фаз осцилляторов, принадлежащих каждому из слоев, так и различающихся на слоях. При дальнейшем увеличении значения $\lambda_{2}$ наблюдается переход к синхронной динамике слоев сети с образованием на слоях идентичных химерных состояний. Распределения фаз осцилляторов для данного случая показаны на рис. 2,c. Видно, что элементы на слоях демонстрируют одинаковую динамику.

Таким образом, в работе впервые изучено взаимодействие химерных состояний в многослойной сети фазовых осцилляторов. Показано, что увеличение степени межслойного взаимодействия не приводит к разрушению химерных состояний. Кроме того, обнаружено, что существуют области значений степени межслойного взаимодействия, при которых возможна реализация как различных, так и общих химерных состояний на слоях сети.

Работа выполнена при поддержке РФФИ (15-02-00624) и Министерства образования и науки РФ (задания 3.861.2017/ПЧ и 3.4593.2017/ВУ).

Письма в ЖТФ, 2017, том 43, вып. 15 


\section{Список литературы}

[1] Abrams D.M., Strogatz S.H. // Phys. Rev. Lett. 2004. V. 93. P. 174102.

[2] Kuramoto Y., Battogtokh D. // Nonlinear Phenom. Complex Syst. 2002. V. 4. P. 380.

[3] Bordyugov G. et al. // Phys. Rev. E. 2010. V. 82. P. 035205.

[4] $G u$ C. et al. // Phys. Rev. Lett. 2013. V. 111. P. 134101.

[5] Sethia G.C. et al. // Phys. Rev. E. 2013. V. 88. P. 042917.

[6] Bera B. et al. // Phys. Rev. E. 2015. V. 93. P. 012205.

[7] Yun Zhu et al. // Phys. Rev. E. 2014. V. 89. P. 022914.

[8] Gambuzza L.V. et al. // Phys. Rev. E. 2014. V. 90. P. 032905.

[9] Tinsley M.R. et al. // Nature Phys. 2012. V. 8. P. 662.

[10] Hagerstrom A. et al. // Nature Phys. 2012. V. 8. P. 658.

[11] Sieber J. et al. // Phys. Rev. Lett. 2014. V. 112. P. 054102.

[12] Levy R. et al. // J. Neurosci. 2000. V. 20. P. 7766.

[13] Motter A.E. // Nature Phys. 2010. V. 6. P. 164.

[14] Panaggio M.J., Abrams D.M. // Nonlinearity. 2015. V. 28. P. R67.

[15] Makarov V.V. et al. // Chaos Soliton. Fract. 2016. V. 84. P. 23.

[16] Sakaguchi H., Kuramoto Y. // Prog. Theor. Phys. 1986. V. 76. P. 576.

[17] Omel'chenko O.E., Wolfrum M. // Phys. Rev. Lett. 2012. V. 109. P. 164101. 